\title{
Das elektrische Fahrzeug als Teilbestand des Energienetzes ${ }^{1}$
}

\author{
G. Spiegelberg
}

Zunächst wird in dem Vortrag auf die Beweggründe eingegangen, warum sich Siemens AG in diesem Umfeld betätigt; einerseits auf der Seite der Infrastruktur für die elektrische Energieverteilung, aber andererseits auch in der Fahrzeugtechnologie.

Ausgehend von dem zunehmenden Eintrag von Energiespitzen ins Netz durch renewable energies sieht Siemens AG seine Kompetenz auf dem Energieverteilsektor für eine Neugestaltung des Netzes, um dieses auch durch bidirektionalen Anschluss des Batteriespeichers von E-Fahrzeugen stabilisieren zu können. Hierdurch kann durch Mehrfachnutzung auch ein Kosten-Sharing der teuren Batterie angedacht werden. Dies erfordert jedoch eine spezielle Ausprägung des fahrzeuginternen Aufbaus und ist daher zur Erreichung von Synergieeffekten nicht unabhängig zu sehen.

Weiterhin wird kurz auf das Gefährdungspotenzial im Automobilsektor eingegangen. Dieser Sektor kann vor allem durch die verringerte Komplexität eines elektrischen Fahrzeugs stark unter Druck geraten, wenn auf einem "Grüne-Wiese"-Ansatz ein Quereinstieg in die Automobiltechnologie von neuen Playern besonders auf dem doch von uns anvisierten Emerging Market vollzogen wird. Hierzu würden drei wesentliche Kriterien einen Ausschlag geben können:

Einerseits die Verringerung der Komplexität des Antriebstrangs durch rein elektrischen Aufbau, zum anderen die Verringerung der Komplexität der Funktionsintegration durch einen völlig neuen An- satz einer elektronischen Systemarchitektur, die modular aufgebaut und generalisiert mit Migrationskonzept über Drive by Wire bis hin zum vollständig autonomen Fahren überführbar sein sollte. Dies kann die Einführung kostengünstiger neuer Fahrzeugkonzepte für die Zukunft unterstützen, die sich an zunehmend verändernden Anforderungen aus den Megatrends "demografischer Wandel, Urbanisierung und environmental Care" orientieren sollten. Unterstützt durch smarte Traffic-Steuerung und Logistikanbindungen kann damit auch ein komfortabler Betrieb von kombiniertem Verkehr entstehen.

Der dritte Punkt wird durch entsprechend günstige, energie- und leistungsstarke Elektrospeicher, also Batterien, gebildet. Hier scheint China mit einer Kapazität von ca. 90 Prozent der gesamten Weltproduktion zumindest kostenseitig Benchmark darzustellen.

Am Ende wird auf das mögliche Potenzial der Fahrzeuganzahl in Deutschland und Europa bis 2020 eingegangen, das erzeugt werden kann, wenn der Markt aktiv bearbeitet wird. Hierzu zählen auch notwendige Maßnahmen in Infrastruktur und Etablierung neuer Geschäftsmodellansätze. Gezeigt wird ein Beispiel aus dem Hause Siemens AG, das in dem Projekt 4Sustain-eMobility das Betreiben einer Testflotte aus Elektrofahrzeugen im Mitarbeiterkreis mit den oben angedeuteten Umfängen beschreibt. 